\title{
PCBP1 and NCOA4 regulate erythroid iron storage and heme biosynthesis
}

\author{
Moon-Suhn Ryu, ${ }^{1}$ Deliang Zhang, ${ }^{2}$ Olga Protchenko, ${ }^{1}$ Minoo Shakoury-Elizeh, ${ }^{1}$ and Caroline C. Philpott ${ }^{1}$ \\ 'Cenetics and Metabolism Section, Liver Diseases Branch, National Institute of Diabetes and Digestive and Kidney Diseases (NIDDK), ${ }^{2}$ Eunice Kennedy Shriver National Institute of Child Health \\ and Human Development, NIH, Bethesda, Maryland, USA.
}

\begin{abstract}
Developing erythrocytes take up exceptionally large amounts of iron, which must be transferred to mitochondria for incorporation into heme. This massive iron flux must be precisely controlled to permit the coordinated synthesis of heme and hemoglobin while avoiding the toxic effects of chemically reactive iron. In cultured animal cells, iron chaperones poly rC-binding protein 1 (PCBP1) and PCBP2 deliver iron to ferritin, the sole cytosolic iron storage protein, and nuclear receptor coactivator 4 (NCOA4) mediates the autophagic turnover of ferritin. The roles of PCBP, ferritin, and NCOA4 in erythroid development remain unclear. Here, we show that PCBP1, NCOA4, and ferritin are critical for murine red cell development. Using a cultured cell model of erythroid differentiation, depletion of PCBP1 or NCOA4 impaired iron trafficking through ferritin, which resulted in reduced heme synthesis, reduced hemoglobin formation, and perturbation of erythroid regulatory systems. Mice lacking Pcbp1 exhibited microcytic anemia and activation of compensatory erythropoiesis via the regulators erythropoietin and erythroferrone. Ex vivo differentiation of erythroid precursors from Pcbp1-deficient mice confirmed defects in ferritin iron flux and heme synthesis. These studies demonstrate the importance of ferritin for the vectorial transfer of imported iron to mitochondria in developing red cells and of PCBP1 and NCOA4 in mediating iron flux through ferritin.
\end{abstract}

\section{Introduction}

Iron deficiency is the most common nutritional deficiency in the world, affecting more than 2 billion people (1). Globally, iron deficiency accounts for 841,000 deaths per year, and the loss of disabilityadjusted life years has been estimated to be above 35 million, primarily due to maternal and perinatal deaths from severe anemia (2). In the adult human, nearly $70 \%$ of total body iron is present in circulating rbc. These cells are produced in the bone marrow, which releases into the bloodstream more than 2 million reticulocytes every second (3). To accomplish this remarkable rate of production, $25 \mathrm{mg}$ of iron per day must be routed to the erythron, taken up into erythroid precursors, delivered to mitochondria, and converted into heme for incorporation into hemoglobin $(\mathrm{Hb})$.

The mechanisms of iron acquisition and utilization by developing rbc have been characterized in considerable detail (4). Upon initiation of development, erythroid progenitors dramatically expand the uptake of transferrin-bound iron $\left(\mathrm{Fe}_{2}-\mathrm{Tf}\right)$ by elevating the expression of transferrin receptors on their cell surface. Iron that is internalized via transferrin receptor-mediated endocytosis is ultimately delivered into mitochondria for heme synthesis as the maturation process progresses. There, iron is incorporated into protoporphyrin IX to form heme, which is eventually delivered to globin proteins to form $\mathrm{Hb}$. Yet the mechanisms by which internalized $\mathrm{Fe}_{2}$-Tf is delivered to mitochondria are poorly understood.

Cytosolic free iron can harm cells due to its chemical reactivity when present in excess or mistrafficked. Thus, the acute expansion

Conflict of interest: The authors have declared that no conflict of interest exists. Submitted: September 6, 2016; Accepted: February 2, 2017. Reference information: J Clin Invest. 2017;127(5):1786-1797. https://doi.org/10.1172/JCI90519. of cellular iron during the late stages of erythropoiesis necessitates a system that can either rapidly deliver the ions into the site of utilization or promptly sequester toxic iron molecules into a storage compartment. Ferritin is the exclusive storage site of elemental iron in the cytosol of mammalian cells. The biologically functional form of ferritin is a 24-subunit, hetero-oligomer composed of 2 peptides, ferritin- $\mathrm{H}$ and ferritin-L, featuring a hollow, spherical cage that can accommodate up to 4,500 iron atoms (5). Ferritin expression rises early in the terminal erythroid differentiation phase and reaches very high levels in parallel with the large amount of iron import in erythroid cells $(6,7)$. However, whether ferritin merely serves to sequester excess iron or plays a more integral role in cellular iron trafficking during maturation is still debated.

Currently, studies suggest 2 routes for the flux of iron from the uptake system to mitochondria: the ferritin-independent and ferritin-dependent paths. Iron-labeling experiments with immature rbcs in circulation suggest that the transfer of iron to mitochondria does not require ferritin (8-10). Instead, these studies propose a direct transfer mechanism in which the iron-laden endosomes directly contact and transfer iron to mitochondria. In support of this hypothesis, a mouse model of ferritin deficiency exhibits relatively normal rbc production, although other iron parameters are highly altered and may have compensatory effects (11). Conversely, ex vivo studies using erythroid progenitor cells demonstrate a transient deposition of exogenous iron into ferritin, which is followed by iron translocation to the heme moiety of $\mathrm{Hb}$ $(12,13)$. Chemical inhibition of lysosome function in these cells leads to a retention of iron in ferritin and impairs incorporation of iron into $\mathrm{Hb}$ (13). These studies indicate that iron must first enter the cytosol and be stored in ferritin and thereafter released from ferritin prior to heme iron synthesis. 
A

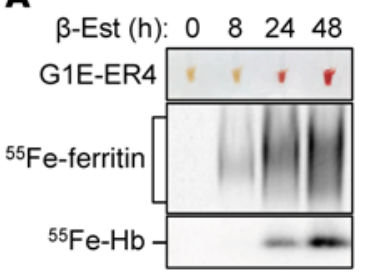

B

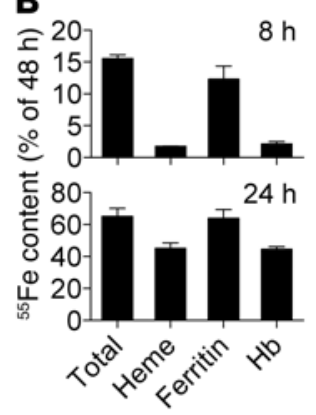

C

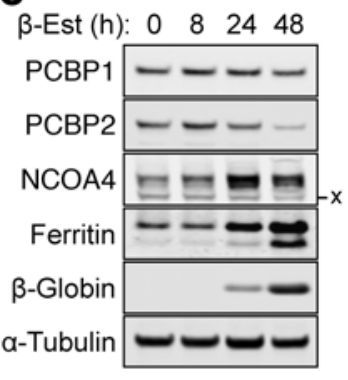

D
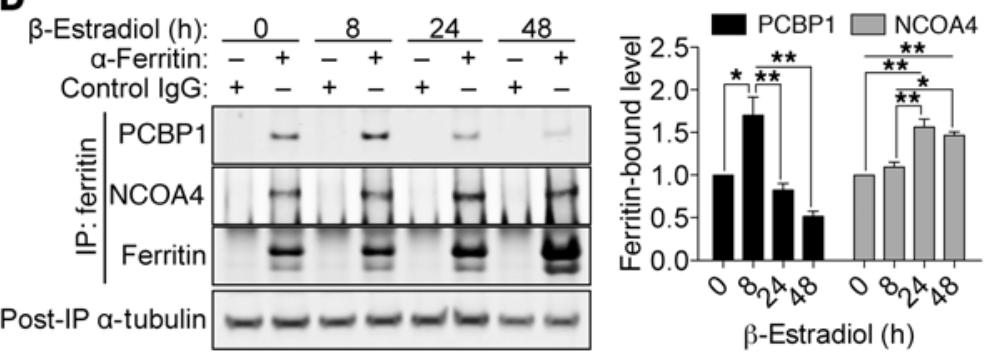

Figure 1. PCBP1 and NCOA4 activities are differentially regulated during rbc maturation in G1E-ER4 cells. Cells were treated with $\beta$-estradiol ( $\beta$-Est), EPO, and ${ }^{55} \mathrm{Fe}_{2}-\mathrm{Tf}$ for 48 hours. (A) Accumulation of ${ }^{55} \mathrm{Fe}$ in ferritin precedes incorporation into $\mathrm{Hb}$ in developing G1E-ER4 cells. Cells harvested at the indicated times were imaged as cell pellets (top) and analyzed by native gel electrophoresis and phosphorimaging. (B) Quantitation of ${ }^{55} \mathrm{Fe}$ accumulation in cells, heme extracts, ferritin, and $\mathrm{Hb}$. Iron levels at 8 hours and 24 hours of development are expressed as percentage of final levels (48 hours). (C) Temporal protein expression patterns of PCBPs and NCOA4 during erythroid maturation. Cells treated as in $\mathbf{A}$ were analyzed by immunoblotting. $X$ indicates nonspecific protein. (D) Developmental regulation of physical interactions between PCBP1 or NCOA4 and ferritin in differentiating erythroid progenitors. Cells treated as in $\mathbf{A}$ were analyzed by quantitative IP of ferritin and immunoblotting. PCBP2 was not detectable in any ferritin precipitates. Ferritinbound PCBP1 and NCOA4 were quantified and normalized to $\alpha$-tubulin in post-IP supernatant. Bulk rabbit IgC included as negative control for nonspecific interactions. $n=3$ independent experiments. ${ }^{*} P<0.05 ;{ }^{* *} P<0.01$, repeated-measures ANOVA with Bonferroni's post-test.

Two proteins, poly r $\underline{C}$-binding protein 1 (PCBP1) and nuclear receptor coactivator $\underline{4}$ (NCOA4) have recently been implicated in controlling the flow of iron into and out of ferritin, respectively, in mammalian cells. PCBP1 is a multifunctional adaptor protein that can bind cellular RNA, DNA, and proteins, altering the fate of its binding partners (14). In erythroid cells, PCBP1 and its close paralog, PCBP2, were found in a nucleoprotein complex that interacts with C-rich sequences in the $3^{\prime}$ UTR of globin transcripts, where they were thought to stabilize the mRNA transcripts prior to translation (15). Subsequent studies identified PCBP1 as an iron-binding protein that delivers iron to ferritin (16) and other iron-dependent proteins, including a mononuclear iron enzyme, deoxyhyppusine hydroxylase (17), and 2 dinuclear iron enzymes, prolyl hydroxylase 2 and asparagyl hydroxylase, that regulate the $\alpha$-subunit of hypoxia-inducible factors (18). This interprotein metal transfer is achieved via direct protein-protein interactions, defining PCBP1 as a cytosolic iron chaperone. Although this metal delivery function is apparent in general cell culture models, especially when cells are iron deficient, whether it is required for or regulated by physiological events in cells specialized for iron trafficking remains unknown.

Recently, 2 independent groups identified NCOA4 as a receptor for autophagic cargo that specifically binds to ferritin and directs it to autophagosomes (19, 20). Ferritincontaining autophagosomes are targeted to lysosomes through autophagosome-lysosome fusion, which results in the degradation of ferritin and the release of its iron core. Cells initiate the turnover of ferritin under iron-deprived conditions, i.e., when the cellular demand for iron is high, by increasing the expression of NCOA4 $(19,21)$. Enhanced gene expression of NCOA4 in cells of erythroid lineage (22) and its dependence on GATA1 (23) have been identified by high-throughput transcriptome analyses, suggesting a role in erythroid maturation. $\mathrm{Hb}$ production is impaired by systemic losses of NCOA4 in zebra fish and mice $(21,24)$ and by NCOA4 RNAi in human cell culture models of erythroid development (21). Yet whether these phenotypes can be attributed to a dysregulation in ferritin iron release during erythroid maturation remains unclear, as NCOA4 has other functions as a transcriptional coactivator involved in various ligand-induced gene activations $(25,26)$ or a repressor of DNA replication (27).

Because the erythron is the major consumer of iron in mammals, we questioned whether PCBP1 and NCOA4 were functioning as ferritin iron regulators in the erythroid system. Here we show that genetic depletion of these proteins leads to erythropoietic defects by inhibiting the cellular flux of iron through ferritin. Our results identify PCBP1 and NCOA4 as indispensable mediators of the vectorial transfer of exogenous iron through ferritin and demonstrate that ferritin is an intermediate source of iron for heme biosynthesis in developing erythroid progenitor cells.

\section{Results}

PCBP1 and NCOA4 differentially interact with ferritin during erythroid maturation. We used G1E-ER4 cells for our in vitro cell culture studies. These cells are an extensively validated murine cell line that recapitulates the early stages of terminal rbc development. G1E-ER4 cells can be induced to differentiate from proerythroblasts into orthochromatic erythroblasts in the presence of erythropoietin (EPO) and $\beta$-estradiol-activated $\operatorname{GATA1}(28,29)$. Morphological changes that accompany differentiation, including nuclear condensation, reduction in cell size, and cytoplasmic polychromatophilia, were reproduced in our G1E-ER4 cells (Supplemental Figure 1A; supplemental material available online with this article; https://doi.org/10.1172/JCI90519DS1). Additionally, we confirmed the transcriptional activation of genes characteristic of erythroid differentiation, including transferrin receptor (Tfrc), erythroid aminolevulinic acid synthase (Alas2), $\alpha$-globin (Hba-a1/2), and $\beta$-globin (Hbb-b1) (Supplemental Figure 1B). In order to track the flow of iron through the cells, we allowed cells to differentiate in the presence of radiolabeled iron. G1E-ER4 cells were induced to differentiate with $\beta$-estradiol for 8,24 , or 48 hours 
A

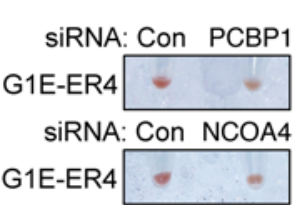

C

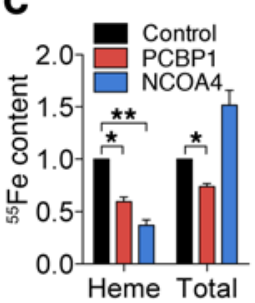

E

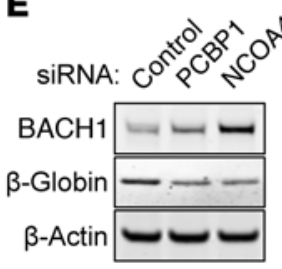

B
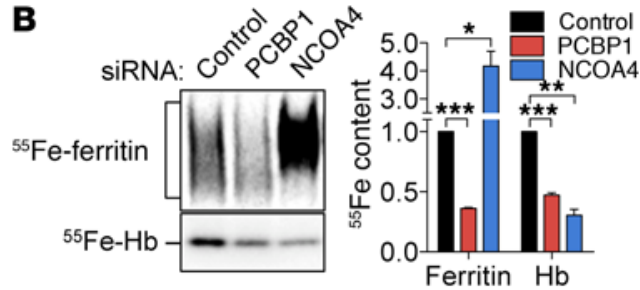

D
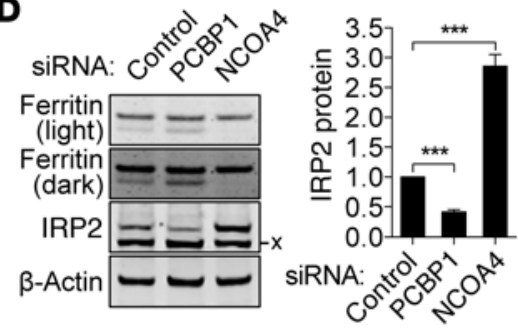

$\mathbf{F}$

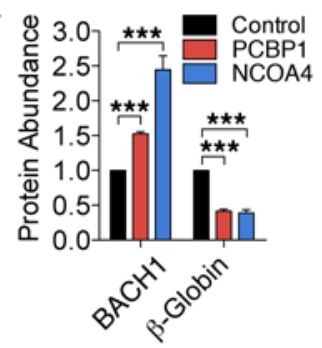

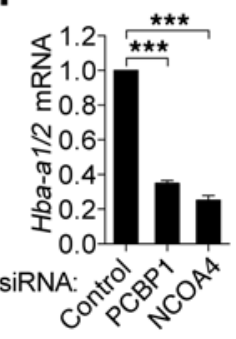

Figure 2. Depletion of PCBP1 or NCOA4 impairs iron flux through ferritin and heme biosynthesis in maturing erythroid progenitors. G1E-ER4 cells were subjected to 2 sequential treatments with a nontargeting control siRNA or with siRNA against PCBP1 or NCOA4, then treated with $\beta$-estradiol and ${ }^{55} \mathrm{Fe}_{2}-\mathrm{Tf}$ for 24 hours. (A) Reduced hemoglobinization of PCBP1- and NCOA4-deficient cells. Representative cell pellets after treatment are shown. Con, control. (B) Altered levels of ${ }^{55} \mathrm{Fe}$ in ferritin and $\mathrm{Hb}$ in PCBP1- and NCOA4-depleted cells. Lysates from cells treated as in $\mathbf{A}$ were analyzed by native gel electrophoresis and phosphorimaging. Quantitation of ${ }^{55} \mathrm{Fe}$ in ferritin and $\mathrm{Hb}$ is shown at right $(n=3)$. (C) Reduced heme synthesis in PCBP1- and NCOA4depleted cells. Levels of ${ }^{55} \mathrm{Fe}$ in heme extracts and whole cells were determined by scintillation counting $(n=3)$. (D) Effects of PCBP1 and NCOA4 depletion on indicators of cellular iron balance. Cells treated as in $\mathbf{A}$ were analyzed by immunoblotting for ferritin and IRP2. X indicates nonspecific protein. IRP2 quantitation shown at right $(n=6)$. (E) Effects of PCBP1 and NCOA4 depletion on indicators of heme levels. Cells were analyzed by immunoblotting for $\mathrm{BACH} 1$ and $\beta$-globin; quantitation at right $(n=6)$. (F) Repression of Hba-a1/2 ( $\alpha$-globin) transcripts in PCBP1- and NCOA4depleted cells. Cells treated as in A were analyzed by real-time PCR (RT-PCR) $(n=5)$. Protein and transcript abundance were normalized to $\beta$-actin and are shown as relative to control siRNA-treated cells. ${ }^{*} P<0.05$; ${ }^{* *} P<0.01$; ${ }^{* *} P<0.001$, repeated-measures ANOVA with Dunnett's test for multiple comparisons with control.

and labeled with ${ }^{55} \mathrm{Fe}$ bound to transferrin $\left({ }^{55} \mathrm{Fe}_{2}-\mathrm{Tf}\right)$, the physiologic iron source for erythroid cells (Figure 1A). Visual inspection of cell pellets demonstrated accumulation of red color, which indicated efficient hemoglobinization over this period. Cell lysates were analyzed by native gel electrophoresis and phosphorimaging to measure the ${ }^{55} \mathrm{Fe}$ incorporated into ferritin and ${ }^{55} \mathrm{Fe}$-heme into $\mathrm{Hb}$. We measured total cellular ${ }^{55} \mathrm{Fe}$ and ${ }^{55} \mathrm{Fe}$-heme by extraction and scintillation counting. Each measurement at 8 and 24 hours was expressed as a percentage of the final level at 48 hours (Figure 1B). Earlier studies suggested that the formation of ferritin iron precedes heme biosynthesis during erythroid maturation $(6,12$, 13). We confirmed that after 8 hours of differentiation, ferritin iron accumulation was readily apparent, while very little iron had been incorporated into heme or $\mathrm{Hb}$. In contrast, after 24 hours of differentiation, iron was substantially present in heme and $\mathrm{Hb}$ as well as in ferritin. These quantitative approaches allowed us to determine that erythroid progenitors rapidly increased iron uptake and ferritin iron storage during differentiation and that iron incorporation into heme and $\mathrm{Hb}$ lagged behind iron uptake and ferritin storage, as previously described $(6,12,13)$.

Proteins involved in heme and $\mathrm{Hb}$ synthesis are upregulated during terminal erythroid differentiation. We measured the abundance of our proteins of interest in the developing G1E-ER4 cells (Figure 1C). While there was no apparent increase in either PCBP1 or PCBP2 levels (PCBP2 levels actually fell), we found a substantial increase in NCOA4, ferritin, and $\beta$-globin expression at later points of maturation. Notably, the increase in NCOA4 production occurred in the stages in which iron incorporation into heme and $\mathrm{Hb}$ was maximal and $\beta$-globin expression was present.

In general cell culture models, both PCBP1 and NCOA4 regulate cellular iron storage through direct protein-protein interaction with ferritin. Thus, the amount of protein bound to ferritin is indicative of their levels of activity. To test whether PCBP1, PCBP2, and NCOA4 bind to ferritin or whether their activities are regulated during erythroid maturation, we quantitatively isolated ferritin from G1E-ER4 cells at different stages of differentiation via IP and measured the amounts of coprecipitated PCBP1, PCBP2, and NCOA4 (Figure 1D). Although levels of PCBP1 in whole cell extracts changed little during differentiation, the amount of PCBP1 in the ferritin immunocomplexes fluctuated, with a peak early in differentiation (8 hours) that fell at later time points. This decrease in coprecipitated PCBP1 occurred despite an increase in total ferritin at later time points. In contrast, PCBP2 was not detectable in any ferritin isolates (data not shown). These data suggested that PCBP1, but not PCBP2, could function as an iron chaperone for ferritin mineralization in erythroid cells undergoing maturation. Ferritin-bound NCOA4 levels correlated well with NCOA4's total cellular expression, featuring higher levels of ferritin-NCOA4 complexes at the later stages of differentiation. These data are consistent with a role for NCOA4 in the mobilization of iron from ferritin when heme synthesis occurs.

PCBP1 and NCOA4 are required for iron storage and hemoglobinization in developing erythroid progenitors. G1E-ER4 cells recapitulated the iron flux previously reported for developing erythroid cells. To characterize the roles of PCBP1 and NCOA4 in this process, we carried out a series of loss-of-function experiments using RNAi in G1E-ER4 cells (Supplemental Figure 2A). Again, we labeled cells with ${ }^{55} \mathrm{Fe}_{2}$-Tf during differentiation. PCBP1- or NCOA4-deficient cells exhibited diminished red coloration after 24 hours of differentiation when compared with controltransfected cells, indicating impaired hemoglobinization (Figure 2A). We hypothesized that this was due to a dysregulation in cellular iron distribution. Phosphorimaging showed a $65 \%$ decrease in ${ }^{55} \mathrm{Fe}$ incorporation into ferritin in cells depleted of PCBP1. Con- 


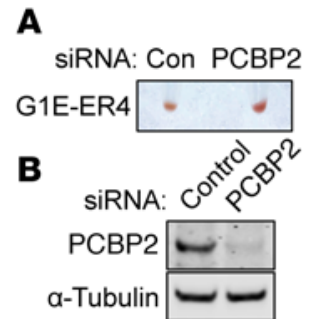

C
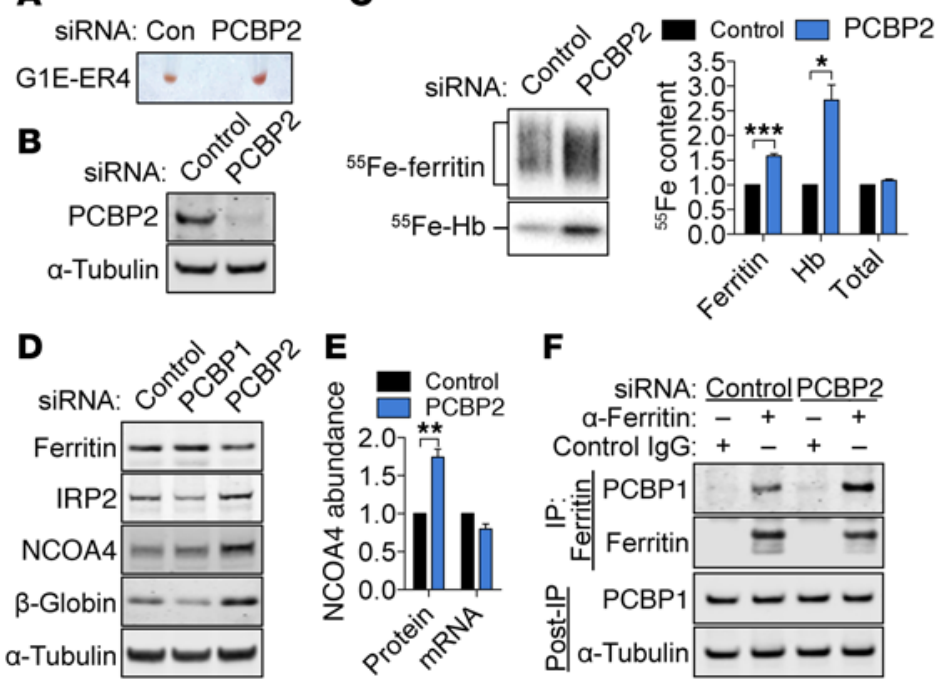

$\mathbf{F}$

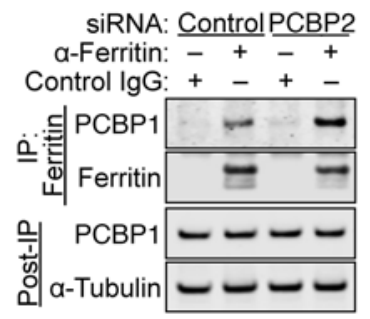

Figure 3. PCBP2 deficiency enhances the flux of exogenous iron through ferritin in developing erythroid progenitors. G1E-ER4 cells were depleted of PCBP2 by siRNA, then treated and analyzed as in Figure 2. (A) Red coloration is more prominent in PCBP2-deficient G1E-ER4 cells. (B) Confirmation of PCBP2 depletion by Western blotting. (C) PCBP2 deficiency increases ${ }^{55} \mathrm{Fe}$ levels in ferritin and $\mathrm{Hb}$ during erythroid development $(n=3)$. (D) PCBP2 depletion affects protein indicators of cellular iron and erythropoiesis in a manner opposite from that of PCBP1 depletion. (E) NCOA4 protein abundance is posttranscriptionally upregulated by PCBP2 depletion $(n=4)$. Transcript and protein abundance were normalized to $\beta$-actin and $\alpha$-tubulin, respectively. (F) PCBP2-depleted cells exhibit increased binding of ferritin to PCBP1 by co-IP. ${ }^{*} P<0.05 ;{ }^{* *} P<0.01$; ${ }^{* *} P<0.001$, paired 2 -tailed $t$ tests.

versely, depletion of NCOA4 resulted in a 4 -fold increase of iron in ferritin (Figure 2B). These data indicate that, in developing rbc, PCBP1 functions as an iron chaperone, facilitating iron accumulation into ferritin, and that NCOA4 is required for iron mobilization from ferritin, consistent with its role as an autophagic cargo receptor. We confirmed the depletion of PCBP1 and NCOA4 to $30 \%$ and less than $10 \%$ of control, respectively, by Western blot analyses (Supplemental Figure 2B).

Although PCBP1 and NCOA4 depletion produced opposing effects on ferritin iron accumulation, both depletions led to lower levels of ${ }^{55} \mathrm{Fe}-\mathrm{Hb}$ (55\% and $70 \%$ reductions, respectively, Figure 2B) and lower levels of heme synthesis (45\% and $65 \%$ reductions, respectively, Figure 2C), as suggested by the loss of red pigmentation. Total iron accumulation was slightly lower in cells differentiating under PCBP1 depletion, but did not account for the lower levels of iron in ferritin or Hb. Partial depletion of PCBP1 produced by half-doses of siRNA also produced defects in iron delivery into ferritin and $\mathrm{Hb}$, yet did not change total cellular iron accumulation (Supplemental Figure 2C). Nontargeting control siRNA did not affect iron uptake or trafficking or erythroid maturation when compared with untransfected cells (Supplemental Figure 2, D-F). Extending the time of differentiation to 48 hours produced defects in $\mathrm{Hb}$ formation that were similar to those seen at 24 hours (Supplemental Figure 3). Wright-Giemsa staining of G1E-ER4 cells after treatment with siRNAs indicated that similar morphological changes associated with differentiation at 24 and 48 hours were present in PCBP1- and NCOA4-depleted cells (Supplemental Figure 4). Taken together, these data indicate that
PCBP1-mediated iron delivery to ferritin and NCOA4mediated iron mobilization from ferritin are critical steps in the flux of iron through the cytosol to the mitochondria of developing G1E-ER4 cells.

PCBP1 and NCOA4 depletion affect regulatory pools of iron and heme. Although we measured large changes in the metallation of ferritin associated with the losses of either PCBP1 or NCOA4, the total ferritin protein levels exhibited small changes that did not parallel the changes in metallation (Figure 2D). Ferritin protein levels tend to increase when cytosolic iron levels rise due to enhanced translation, which is controlled by the iron regulatory protein-iron responsive element (IRP-IRE) regulatory system (30). Iron regulatory protein 2 (IRP2) is targeted for degradation when iron levels rise, which relieves the IRP2-mediated block on ferritin translation. PCBP1 deficiency produced a 63\% decrease in IRP2 protein abundance, suggesting an expansion in the cytosolic iron pool and enhanced degradation of IRP2. In contrast, NCOA4 depletion produced a $180 \%$ increase in IRP2, consistent with a decrease in cytosolic iron levels and IRP2 degradation, likely due to ferritin sequestration of iron. These changes in IRP2 indicate that the cytosolic labile iron pool was affected by impaired loading and release of iron from ferritin caused by depletion of PCBP1 and NCOA4, respectively. Ferritin protein levels are also regulated via autophagic turnover, mediated by $\operatorname{NCOA} 4(19,20)$. Autolysosomal degradation of ferritin is associated with the appearance of a lower molecular weight, truncated form of ferritin (20). This form was apparent in control cells and increased in PCBP1-depleted cells, but not present in NCOA4-depleted cells (Figure 2D). The changes in total ferritin protein levels were small, likely because changes in ferritin translation were offset by concomitant changes in ferritin turnover.

Erythroid cells express multiple regulatory systems to avoid excess globin production under conditions of diminished heme production. We questioned whether the heme deficiency produced by PCBP1 and NCOA4 depletion resulted in altered regulation of globin production. BTB domain and $\mathrm{CNC}$ homolog 1 (BACH1) is an erythroid transcriptional repressor that is rapidly degraded under conditions of heme sufficiency (31). We observed that BACH1 levels were elevated and that transcripts of $\mathrm{Hba-a1/2}$ ( $\alpha$-globin), which are targets of BACH1 (32), were repressed in PCBP1- and NCOA4-depleted cells (Figure 2, E and F). PCBP1 and NCOA4 depletion also resulted in lower $\beta$-globin protein levels, although changes in $H b b$ - $b 1$ mRNA were much smaller (Supplemental Figure $5 \mathrm{~A}$ ). In contrast, transcript levels of Alas2, the rate-limiting enzyme for heme biosynthesis, were increased in cells depleted of PCBP1 or NCOA4. These data indicate that the heme deficiency associated with PCBP1 or NCOA4 depletion altered the regulatory systems designed to coordinate heme and globin synthesis.

Iron supplementation fails to restore Hb levels in PCBP1- and NCOA4-depleted cells. We questioned whether the requirement for iron flux through ferritin was critical for erythroid maturation or whether the requirement could be bypassed by iron supplementation. Therefore, we depleted G1E-ER4 cells of PCBP1 or NCOA4 and allowed them to differentiate for 24 or 48 hours in the presence or absence of supplemental iron. Treatment of cells with iron 

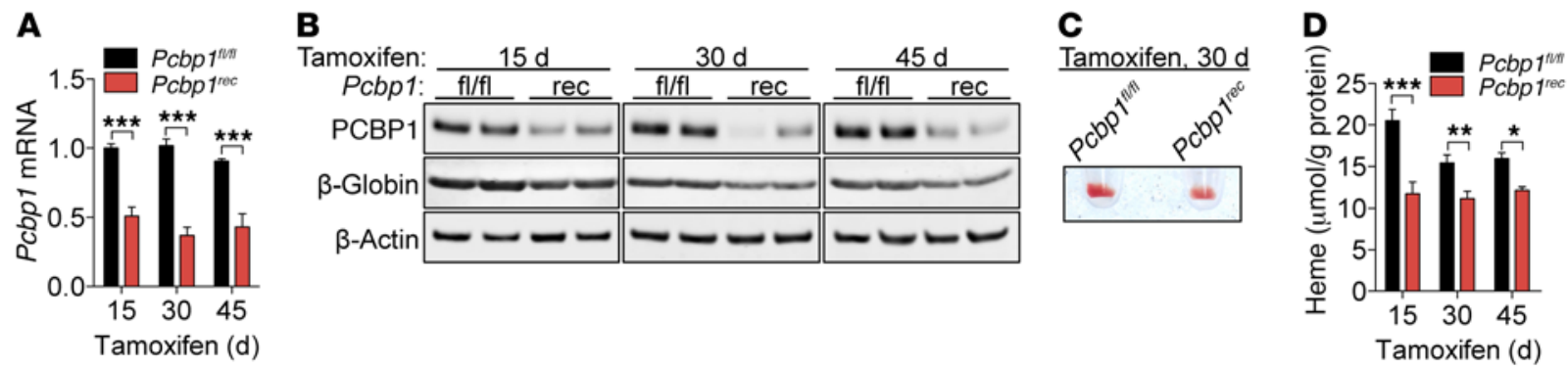

Figure 4. Pcbp1 is required for erythroid heme and globin production in mice. A murine model of $P c b p 1$ deficiency was generated using a tamoxifeninducible Cre recombinase-loxP system $\left(P c b p f^{f / f l} C r e-E R^{T}\right)$. Male $P c b p f^{f / f f}$ and $P c b p 7^{f l / f l} C r e-E R^{T}$ mice were weaned onto a tamoxifen diet for 15,30 , or 45 days to produce PCBP1-deficient $\left(P c b p 1^{r e c}\right.$ ) and control animals $\left(P c b p 7^{f / f f}\right)$, respectively. Bone marrow was collected and analyzed by quantitative RT-PCR and immunoblot. (A) Loss of PCBP1 transcripts in bone marrow by tamoxifen-induced recombination (Pcbp $7^{f / f f}, n=5 ; P c b p 7^{\text {rec }}, n=3-6$ per group). (B) Reduced $\beta$-globin protein in the bone marrow of Pcbp1-deficient animals. (C) Lower Hb pigment in Pcbp1-deficient bone marrow cells. (D) Lower heme levels in bone marrow of Pcbp1-deficient mice. $n=5 P c b p 7^{f l / f l} ; n=3-6 P c b p{ }^{r e c}$ per time point. ${ }^{*} P<0.05 ;{ }^{* *} P<0.01 ;{ }^{* *} P<0.001$ by 2 -way ANOVA with Bonferroni's post-test.

for 4 or 24 hours produced a time-dependent increase in ferritin protein levels in control and PCBP1-depleted cells, consistent with an iron-mediated increase in ferritin translation, confirming the uptake of supplemental iron (Supplemental Figure 5, B and C). The increased iron did not restore $\beta$-globin levels in PCBP1depleted cells, however, suggesting that the defect in heme synthesis persisted. Similarly, supplemental iron did not correct the $\beta$-globin deficit of NCOA4-depleted cells. As the translational upregulation of ferritin was also blocked in the NCOA4-deficient cells, these data suggest the supplemental iron was sequestered in ferritin. Taking these data together, we demonstrate that the iron flux through ferritin is an obligate component of mitochondrial heme synthesis in early to intermediate stages of terminal differentiation regardless of cellular iron availability.

Loss of PCBP2 enhances the flux of iron through ferritin. The above loss-of-function experiments demonstrated the requirement of PCBP1 for the entrance of iron into ferritin in developing erythroid cells. The PCBP protein family is composed of 4 members, all of which have been implicated in controlling cytosolic iron distribution $(16,33)$. Although PCBP2 was not detectable in ferritin immunoprecipitates, it was highly expressed in developing G1EER4 cells (Figure 1C). This led us to test whether PCBP2 affects cellular iron during rbc maturation. Again, we used siRNA to deplete cells of PCBP2 and ${ }^{55} \mathrm{Fe}_{2}$-Tf labeling to track iron flux in G1E-ER4 cells. Surprisingly, cells exhibited enhanced hemoglobinization when depleted of PCBP2 (Figure 3, A and B, and Supplemental Figure 3, A and B). ${ }^{55} \mathrm{Fe}$-labeling revealed that PCBP2-depleted cells exhibited a 50\% increase in iron incorporation into ferritin and a $150 \%$ increase into $\mathrm{Hb}$, without a change in total iron accumulation (Figure 3C). Measurement of protein abundance also revealed responses opposite of those associated with PCBP1 deficiency, as levels of IRP2, $\beta$-globin, and NCOA4 all increased by PCBP2 depletion (Figure 3D, Supplemental Figure 3D, and Supplemental Figure 6A). Each of these changes in protein levels was due to posttranscriptional mechanisms, as mRNA levels, including Alas2, did not change (Figure 3E and Supplemental Figure 6B). Hba-a1/2 transcript levels increased with PCBP2 depletion, although BACH1 protein levels did not change (Supplemental Figure 6, B and C).

We questioned whether the increase in ferritin iron associated with PCBP2 deficiency could be due to increased activity of
PCBP1. Accordingly, we measured ferritin-bound PCBP1 levels after depletion of PCBP2. The loss of PCBP2 resulted in elevated ferritin-bound PCBP1, without changes in its total protein level (Figure 3F). If the effects of PCBP2 deficiency on ferritin iron loading were mediated through PCBP1, then cells depleted of both PCBP2 and PCBP1 should phenotypically resemble cells lacking PCBP1 alone. We found that simultaneous depletion of PCBP1 and PCBP2 produced a defect in ferritin iron loading identical to that of cells lacking PCBP1 alone (Supplemental Figure 6, D and E). These data demonstrate that PCBP2 suppressed the iron chaperone activity of its homolog, PCBP1, in ferritin mineralization. Notably, $\mathrm{Hb}$ iron contents were slightly higher in cells with the simultaneous loss of PCBP1 and PCBP2 when compared with those deficient in PCBP1 only. This implies that iron may reach mitochondria for heme synthesis independently of PCBP1, possibly through enhanced transfer of ferritin iron to the lysosome via NCOA4-mediated ferritinophagy.

Conditional PCBP1 deficiency in mice leads to microcytic anemia. Consistent with our cell-based studies, mouse models of NCOA4 deficiency are associated with defects in rbc hemoglobinization $(21,24)$. The role of PCBP1 in erythropoiesis has not been studied in a live animal model; therefore, we tested to determine whether loss of PCBP1 activity in mice could lead to erythropoietic defects. $P c b p 1$ is indispensable for murine embryonic development (34). To circumvent the lethality of constitutive germline Pcbp1 deletion, we generated a conditional deletion model in which the Pcbp1 locus is flanked by loxP sites $\left(P c b p 1^{f / f}\right)$. By breeding the Pcbp $1^{1 / f l}$ mice into a tamoxifen-inducible Cre recombinase background $\left(C r e-E R^{T}\right)$, we were able to produce $P c b p 1^{f / f l}$ and $P c b p 1^{f / f l} C r e-E R^{T}$ mice (Supplemental Figure 7). Recombination was induced by weaning mice onto a tamoxifen diet for 15,30 , and 45 days prior to phenotypic analyses.

We measured PCBP1 transcript and protein abundance in bone marrow cells after tamoxifen induction and found that abundance was decreased in the Cre-ER ${ }^{\mathrm{T}}$-expressing mice (Pcbp1 $1^{r c}$ ) to $40 \%-50 \%$ of the levels in $P c b p 1^{1 / f l}$ mice, suggesting a moderate level of recombination efficiency (Figure 4, A and B). To determine the level of recombination in erythroid progenitors in the bone marrow, we isolated Ter119+ ${ }^{+}$cells from $P c b p 1^{f / / l}$ and Pcbp1 $1^{\text {rec }}$ mice after tamoxifen treatment and measured a $70 \%-$ 
A

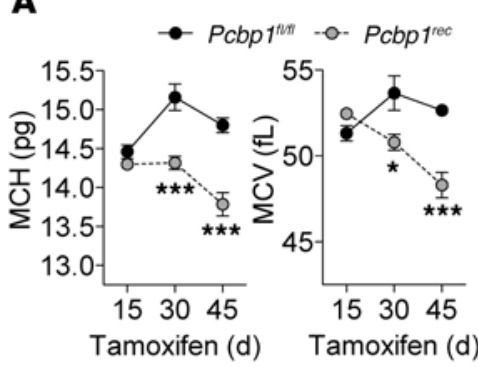

B

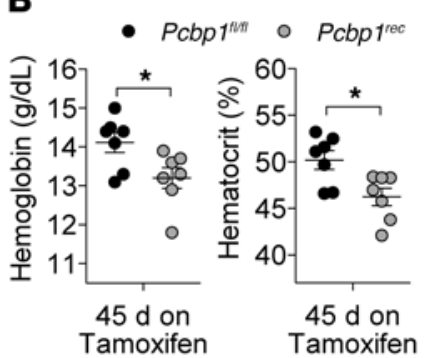

C

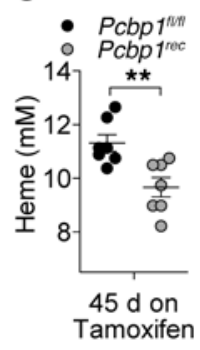

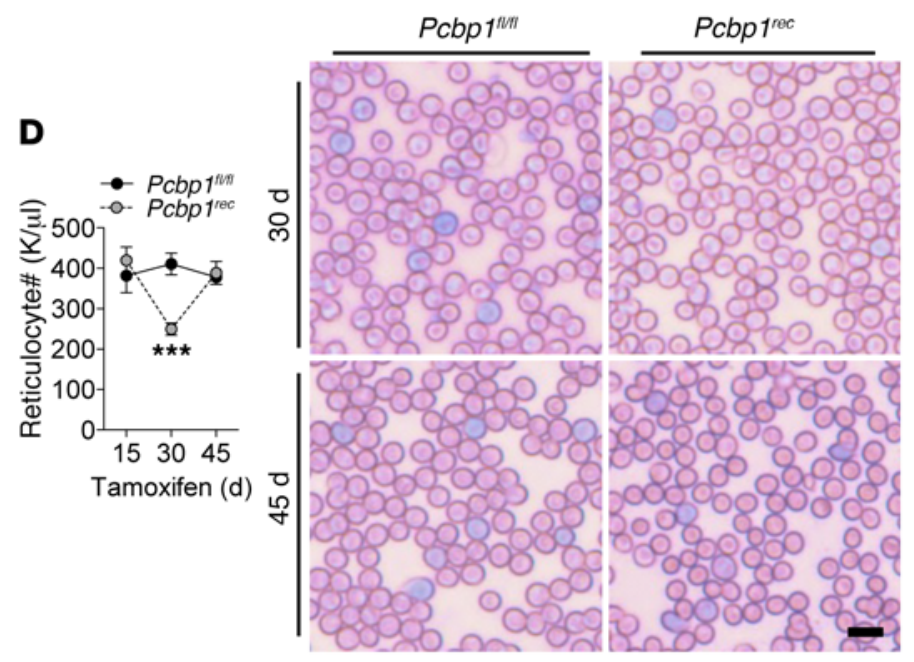

Figure 5. Prolonged Pcbp1 deficiency produces microcytic anemia. Whole blood from Pcbp $1^{f / f l}$ and Pcbprec mice treated with tamoxifen as in Figure 4 was collected and analyzed. (A) Development of heme-deficient and microcytic rbc in Pcbprec mice after 30 and 45 days of tamoxifen. (B) Reduced $\mathrm{Hb}$ and hematocrit in Pcbprec mice after 45 days of tamoxifen. (C) Reduced whole blood heme levels in $P c b p r^{r e c}$ mice after 45 days of tamoxifen. (D) Transient reduction in circulating reticulocytes after 30 days of tamoxifen measured by CBC (left) and Wright-Giemsa staining of blood smears (right). Scale bar: $10 \mu \mathrm{m} . n=5-7$ Pcbp1fl/f;; 3-7 Pcbp1rec per time point. ${ }^{*} P<0.05 ;{ }^{* *} P<0.01$; ${ }^{* *} P<0.001,2$-way ANOVA with Bonferroni's post-test (A and $\mathbf{D}$ ) or 2-tailed $t$ tests (B and $\mathbf{C}$ ).

tion width (RDW) was increased by 45 days in Pcbp1 $^{\text {rec }}$ (Supplemental Table 1). These changes in erythrocyte indices reflected progressive development of microcytotic anemia in PCBP1-deficient mice and resembled the prototypical manifestations of iron deficiency anemia. Consistent with iron-limited erythropoiesis, $P c b p 1^{r e c}$ mice at 30 days exhibited a precipitous loss of circulating reticulocytes (56\% of $P c b p 1^{f l / f l}$, Figure 5D), which increased to control levels by 45 days. Wright-Giemsa stains of blood smears also demonstrated a loss of reticulocytes at day 30 , followed by recovery of reticulocyte production by day 45 . These changes suggested a potential compensatory response triggered by the anemia due to PCBP1 deficiency.

Compensatory erythropoiesis accompanies anemia in Pcbp1 deficiency. Reticulocyte numbers reflect the capacity of erythropoietic tissues to increase production of immature rbc to meet the demands of the animal. Histological analysis of bone marrow sections from both $P c b p 1^{r e c}$ and $P c b p 1^{f l / f l}$ mice at day 30 revealed a highly cellular marrow with nucleated cells of multiple lineages present. Assay for apoptotic cells revealed no differences between genotypes (Supplemental Figure 8B), indicating the absence of acute bone marrow failure and suggesting that the reduction in reticulocyte release from the erythropoietic tissue was not due to apoptotic loss of erythroid progenitor cells.

The increase in reticulocyte number detected at day 45 in the $P c b p 1^{r e c}$ mice sug-

75\% decrease in PCBP1 transcript levels (Supplemental Figure 8A). The partial depletion of $P c b p 1$ was not enhanced by longer periods of dietary tamoxifen. Bone marrow cells from $P c b p 1^{r e c}$ mice featured $30 \%-35 \%$ lower $\beta$-globin protein levels and less intense red coloration, suggesting defects in hemoglobinization of the erythropoietic cells (Figure 4, B and C). Direct measurement of heme content indicated that Pcbp1 ${ }^{r e c}$ mice exhibited $25 \%-40 \%$ lower heme levels in the bone marrow compared with $P c b p 1^{f / f l}$ animals (Figure 4D). These data suggested a defect in erythroid cell maturation in PCBP1-deficient bone marrow.

Although the erythropoietic defects in bone marrow cells of Pcbp $1^{\text {rec }}$ mice occurred within 15 days of tamoxifen treatment, rbc parameters of circulating whole blood appeared relatively spared during this early stage of PCBP1 deficiency. After 30 days of tamoxifen, however, PCBP1-deficient animals exhibited hematological changes as measured by complete blood counts (CBC) (Figure 5 and Supplemental Table 1). The mean corpuscular $\mathrm{Hb}(\mathrm{MCH})$ and mean corpuscular volume (MCV) were significantly lower in the PCBP1-deficient mice when compared with wild-type controls (Figure 5A). By day 45, Pcbp1 ${ }^{\text {rec }}$ mice additionally exhibited significant decreases in total $\mathrm{Hb}$ and hematocrit (Figure $5 \mathrm{~B}$ ) and in the total heme content of whole blood (Figure 5C). Red cell distribu- gested a compensatory response to the anemia. Anemia is sensed in the renal cortex as a relative hypoxia, which triggers the synthesis and release of EPO. We measured the Epo transcripts in the kidneys of $P c b p 1^{t / f l}$ and $P c b p 1^{r e c}$ mice and found that, at 30 days of tamoxifen, levels were similar, but at 45 days of tamoxifen, $P c b p 1^{\text {rec }}$ mice exhibited a 2-fold increase in Epo transcript levels (Figure 6A). PCBP1 deficiency was detected in kidney at both 30 and 45 days of tamoxifen, suggesting that the Epo response was not primarily due to PCBP1 deficiency in the kidney. Recent work has identified erythroferrone (ERFE, Fam132b) as an erythroid factor that responds to the EPO released under conditions of stimulated erythropoiesis (35). We measured Erfe transcripts in the bone marrow of $P c b p 1^{f l / f l}$ and $P c b p 1^{\text {rec }}$ mice and again found that Erfe transcript levels were similar at days 15 and 30, but that levels were 3-fold higher in the Pcbp1 ${ }^{\text {rec }}$ mice after 45 days (Figure 6B). ERFE is released from the bone marrow and sensed in the liver, where it functions to transcriptionally repress the expression of hepcidin (HAMP), the major regulator of dietary iron assimilation and macrophage iron release. We measured Hamp transcripts in the liver and found that, at day 45 , their levels were reduced by $70 \%$ in the Pcbp1 ${ }^{\text {rec }}$ mice (Figure 6C). These data all indicate that prolonged PCBP1 deficiency was associated with iron-limited anemia, which 
A
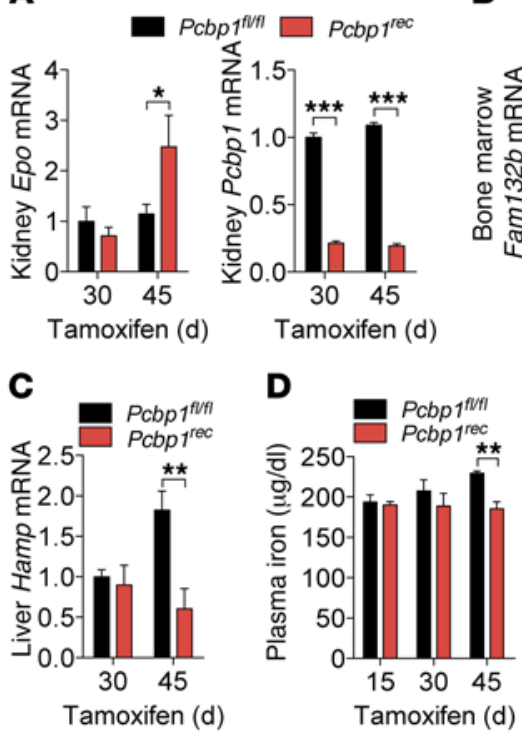

B
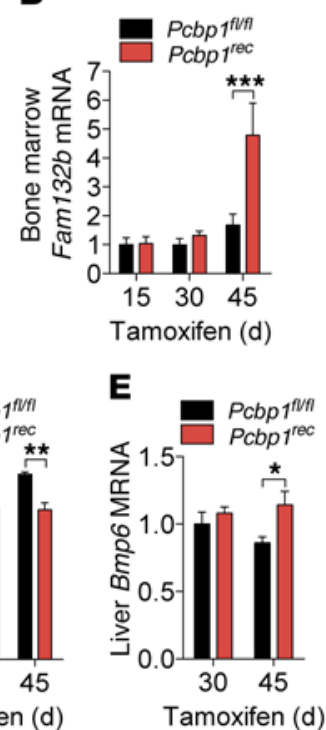

Figure 6. Compensatory responses to anemia in Pcbp1-deficient animals. Tissues from $P c b p 7^{f / f l}$ and $P c b p 7^{r e c}$ mice treated with tamoxifen as in Figure 4 were harvested and analyzed by quantitative RT-PCR, nonheme iron measurement, or histochemistry. (A) Increased Epo mRNA levels in kidney of Pcbp1 rec mice at 45 days. Depletion of PCBP1 was measured at 30 and 45 days. (B) Elevated Fam132b (ERFE) transcript abundance in bone marrow of Pcbp $7^{\text {rec }}$ mice at 45 days. (C) Repression of Hamp (hepcidin) expression at 45 days in Pcbp $7^{r e c}$ mice. (D) Small decrease in plasma iron levels in Pcbprec mice. Plasma was collected and nonheme iron levels were measured. (E) Small increase in liver $B m p 6$ transcripts not accounting for change in hepcidin. $n=5-7$ Pcbp $7^{f / f f} ; n=3-7 P c b p 1^{\text {rec }}$ per time point. ${ }^{*} P<0.05$; ${ }^{* *} P<0.01$; ${ }^{* * *} P<0.001,2$-way ANOVA with Bonferroni's post-test.

activated the EPO-ERFE-hepcidin regulatory axis for compensatory iron uptake and rbc production.

Perturbations in systemic iron homeostasis can also affect Hamp gene expression through regulation of bone morphogenetic protein 6 (BMP6) (36). We measured plasma iron levels in Pcbp $1^{1 / / 7}$ and $P c b p 1^{\text {rec }}$ mice and observed a small but significant decrease in plasma iron levels in PCBP1-deficient mice on day 45 (Figure 6D). The decrease in plasma iron was not associated with a decrease in Bmp6 transcripts, however, but rather a small increase (Figure $6 \mathrm{E})$. These data suggest that the repression in liver Hamp expression was not primarily due to the changes in circulating iron, as these would be expected to decrease, rather than increase, expression of Bmp6. Similarly, Pcbp1 recombination in the liver did not account for the repression in Hamp (Supplemental Figure 9). Thus, hepcidin repression was likely a consequence of elevated ERFE production. Collectively, we identified microcytic anemia as a phenotypic outcome of PCBP1 deficiency that leads to compensatory erythropoiesis via EPO-mediated Erfe activation.

PCBP1-deficient primary erythroid progenitors develop with impaired iron delivery to ferritin and $\mathrm{Hb}$ ex vivo. Our animal model of PCBP1 deficiency is complicated by variable degrees of PCBP1 recombination in other, nonerythropoietic tissues. To isolate the effects of PCBP1 deficiency in erythropoietic tissues, we assessed the iron utilization and maturation of isolated primary erythroid progenitors from $P c b p 1^{f / f l}$ and $P c b p 1^{\text {rec }}$ mice ex vivo. Phenylhydrazine (PHZ) treatment causes acute hemolytic anemia in mice, which transforms the spleen into the primary site of compensatory erythropoiesis. Using this well-established model, we harvested spleens from PHZ-challenged mice and isolated erythroid progenitors, synchronized at the CFU-erythroid (CFU-E) stage of committed differentiation (37). Pcbp $1^{\text {rec }}$ mice had smaller spleens than $P c b p 1^{1 / f / l}$ mice and exhibited a $60 \%$ lower recovery of nucleated erythroid cells, although recovered cells exhibited no differences in viability (Figure 7A). Erythroid splenocytes from PCBP1-deficient animals were pale, with less red coloration and less $\beta$-globin protein than in $P c b p 1^{f / f l}$ animals (Figure 7B), implying less effective stress erythropoiesis and recovery from hemolytic anemia in the $P c b p 1^{\text {rec }}$ mice.

We cultured the isolated splenocytes in the presence of EPO and 4-hydroxytamoxifen to promote ex vivo differentiation and sustained recombinase activity, respectively. $\beta$-Globin protein expression was increased after 20 hours of EPO treatment in both $P c b p 1^{f / f l}$ and $P c b p 1^{r e c}$ mice, confirming ex vivo differentiation (Figure 7C), although $\beta$-globin levels were lower in PCBP1-deficient cells than in wild-type cells throughout the time course. Ferritin and NCOA4 protein levels decreased and increased, respectively, in both genotypes. Radiolabeling with ${ }^{55} \mathrm{Fe}_{2}-\mathrm{Tf}$ during differentiation identified a clear impairment in ferritin metallation in the erythroid progenitors from PCBP1-deficient mice at both 8 and 20 hours (Figure 7D). We also observed a $43 \%$ decrease in iron incorporation into $\mathrm{Hb}$ after 20 hours of differentiation in the Pcbp1 $1^{\text {rec }}$ mice. The reduced incorporation of iron into ferritin and $\mathrm{Hb}$ was not attributable to changes in total cellular iron accumulation during ex vivo development (Figure 7E). These observations support our findings from the in vitro and in vivo experiments indicating that the iron chaperone activity of PCBP1 is required for erythroid ferritin mineralization, which is an obligatory step in iron trafficking to mitochondria for heme biosynthesis in maturing rbc.

\section{Discussion}

In general cell culture systems, mammalian cells exhibit a requirement for iron chaperone activity, mainly under conditions of iron limitation $(16-18,33)$. Whether animals or tissues and cells that are specialized for iron transport and utilization also depend on iron chaperones was not known. Our studies, using data derived from cultured cell, whole animal, and ex vivo differentiation models, all indicate that the iron chaperone activity of PCBP1 is required for the efficient use of iron by developing erythropoietic cells. Furthermore, we demonstrate that PCBP1 and NCOA4 are necessary for the flow of iron through ferritin in these cells and that supplemental iron does not rescue the defects associated with depletion. Thus, the PCBP1-dependent transfer of iron into ferritin and the NCOA4-dependent transfer of iron out of ferritin are critical processes for efficient iron utilization in erythropoiesis. Our data also suggest that the relative contributions of PCBP1 and NCOA4 to iron flux through ferritin change over the course of erythroid development (Figure 8). In our model, the earliest stage of terminal differentiation is characterized by iron accumulation, which precedes heme and Hb synthesis. PCBP1 activity for ferritin is high, and ferritin turnover in the lysosome is low. As differentiation progresses, iron uptake and PCBP1-mediated iron delivery to ferritin continue, but NCOA4-mediated recruitment of ferritin to the autophagosome also occurs, as does iron transfer 
A

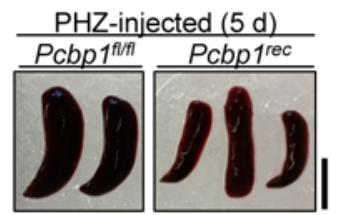

Cells: $\left.147.6 \pm 18.760 .3 \pm 8.3^{*} \times 10^{6}\right)$ Viability: $87.8 \pm 1.0 \quad 91.1 \pm 1.4(\%)$
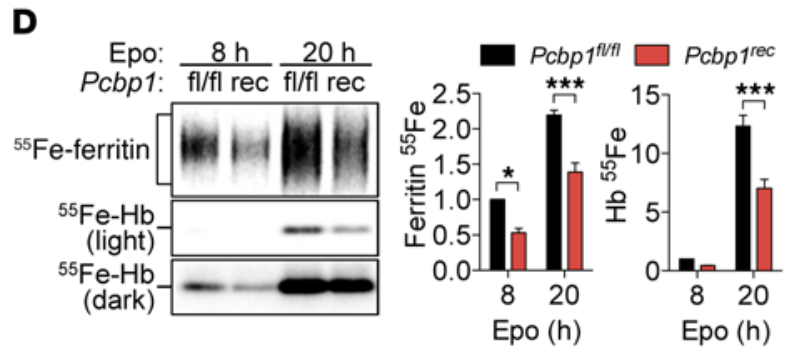
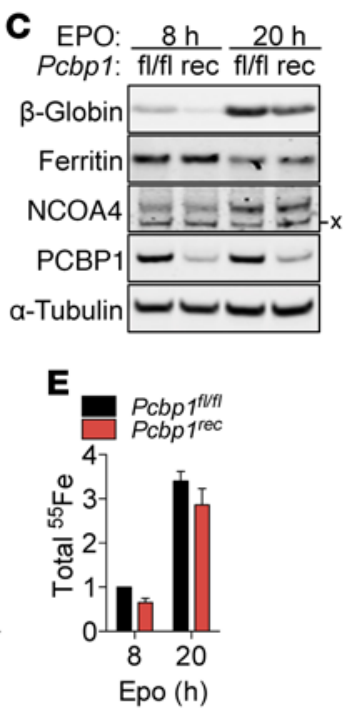

Figure 7. Primary erythroid progenitors from Pcbp1-deficient mice exhibit impaired flux of iron through ferritin during ex vivo maturation. Primary erythroid progenitors were prepared from

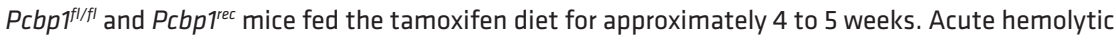
anemia was produced by i.p. injections of $\mathrm{PHZ}$ ( $60 \mathrm{mg} / \mathrm{kg}$ body weight) on days 1 and 2 , and spleens were harvested on day 5. (A) Reduced spleen size and cellular content in PCBP1-deficient mice. (B) Reduced Hb pigment and $\beta$-globin protein in splenocytes from PHZ-treated Pcbp1-deficient mice versus controls. (C-E) Ex vivo differentiation of isolated splenic erythroid progenitors. Splenocytes were treated with EPO $(2 \mathrm{IU} / \mathrm{ml})$ and ${ }^{55} \mathrm{Fe}_{2}$-Tf for the indicated times. (C) Lower $\beta$-globin protein expression in Pcbp1-deficient primary erythroid cells undergoing ex vivo maturation. $\mathrm{X}$ indicates nonspecific protein. (D) Impaired transfer of exogenous iron into ferritin and $\mathrm{Hb}$ during ex vivo development of Pcbp1-deficient primary erythroid cells. (E) Pcbp1 genotype did not affect total ${ }^{55} \mathrm{Fe}$ accumulation. $n=3$ independent experiments. ${ }^{*} P<0.05 ;{ }^{* * *} P<0.001$, repeated measures ANOVA with Bonferroni's post-test.

from lysosomes to mitochondria. Rates of heme synthesis and $\mathrm{Hb}$ formation are high. In the latest stage of differentiation, PCBP1mediated iron delivery to ferritin is low, iron delivery to mitochondria may include direct transfer from endosomes, and the last fractions of heme and $\mathrm{Hb}$ are synthesized before organelles are ejected and the circulating reticulocyte takes its mature form.

Depletion of PCBP1 or NCOA4 in erythroid cells appears to uncouple ferritin iron accumulation from the IRP-IRE regulatory system. IRP1 and IRP2 are part of a cell-autonomous, posttranscriptional, iron regulatory system in which the cytosolic labile iron pool is sensed and the IRPs respond by altering the synthesis of proteins involved in iron homeostasis (30). IRP1 and IRP2 repress the translation of ferritin mRNA when cytosolic iron is low. As iron levels rise, IRP1 and IRP2 RNA-binding activity is progressively lost and ferritin protein is synthesized. Typically, iron accumulation in ferritin parallels ferritin protein synthesis. In PCBP1-depleted G1E-ER4 cells, IRP regulation appears intact; iron supplementation leads to increased ferritin protein synthesis. PCBP1 depletion also promoted a decrease in the levels of IRP2, the degradation of which is triggered by elevated cytosolic iron (30). Yet iron accumulation into ferritin is drastically impaired. Reciprocally, in NCOA4-depleted cells, ferritin protein levels were comparable to control levels, a somewhat paradoxical effect for a protein that mediates ferritin turnover. In this instance, it appears that iron accumulation in ferritin triggers cytosolic iron depletion and concomitant activation of RNA binding by IRP1 and IRP2. IRP binding leads to repression of ferritin synthesis, even under iron supplementation. However,

PCBP1-mediated loading of iron into ferritin continues unabated, leading to large increases in ferritin iron without increases in ferritin protein. Thus, despite appropriate iron sensing by the IRP-IRE system, ferritin iron accumulation becomes uncoupled from ferritin protein levels. In a sense, PCBP1 and NCOA4 are essential components of the cell-autonomous iron regulatory system.

Ferritin is the only cytosolic storage protein for elemental iron identified in mammalian cells. Because excess cytosolic iron is toxic, some investigators hypothesize that the primary function of erythroid ferritin is the removal and sequestration of surplus iron from the cytosol and the mitochondrial heme-producing machinery (8-10). Our work confirms an alternative hypothesis that identifies ferritin as an indispensable intermediate in the transfer of acquired iron between the transferrin-iron uptake machinery and the mitochondria $(12,13)$. These studies were carried out with G1E-ER4 cells at an intermediate stage of terminal development, from proerythroblasts to basophilic erythroblasts; the ex vivo studies were carried out with splenic erythroid precursors synchronized at the CFU-E stage. Both approaches reveal the early stages of committed erythropoiesis as the developmental period requiring coordinated PCBP1 and NCOA4 activity and their common target, ferritin, for efficient iron utilization. Our whole animal studies indicate that disruption of efficient iron utilization by PCBP1 depletion ultimately leads to microcytic anemia, underscoring the importance of these early events in erythroid iron utilization.

The role of ferritin in early erythroid development does not negate evidence for ferritin-independent iron trafficking in late stages of development (8-10). Reticulocytes that are newly released from the bone marrow contain RNA, ribosomes, mitochondria, and lysosomes (38). After release into the circulation, these early reticulocytes further mature, ejecting their intracellular organelles, e.g., mitochondria and lysosomes. Early reticulocytes actively synthesize heme and $\mathrm{Hb}$, but deposit very little iron in ferritin (6). Thus, the role of ferritin in erythrocyte iron flux may be limited to the earliest stages of rbc development. The interaction of PCBP1 with ferritin is greatly lessened in the late stages of development in G1E-ER4 cells, consistent with a diminishing role of ferritin iron transit as erythrocytes mature. The mechanism that accounts for this change in PCBP1-ferritin interaction remains to be investigated. Although mice with partial depletion of PCBP1 or systemic Ncoa4 knockout exhibit microcytic anemia (24), mice with conditional deletion of ferritin $\mathrm{H}$ do not (11). The reason for this difference is not yet identified and may be multifactorial. Mice with ferritin $\mathrm{H}$ deletion exhibit increased transferrin saturation and serum iron; they may exhibit other alterations in iron balance, such as circulating non-transferrin-bound iron. These changes may serve to increase iron delivery to the erythron and compen- 
A

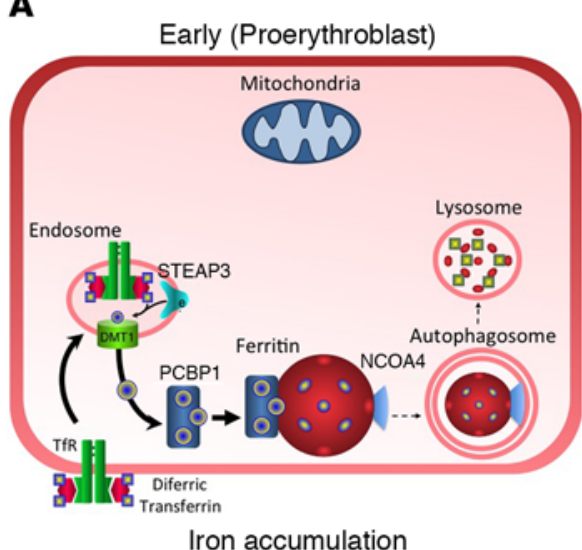

B

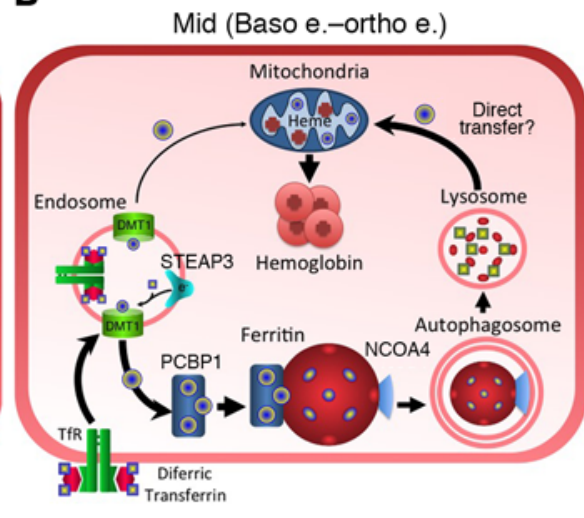

Iron accumulation; heme and globin synthesis

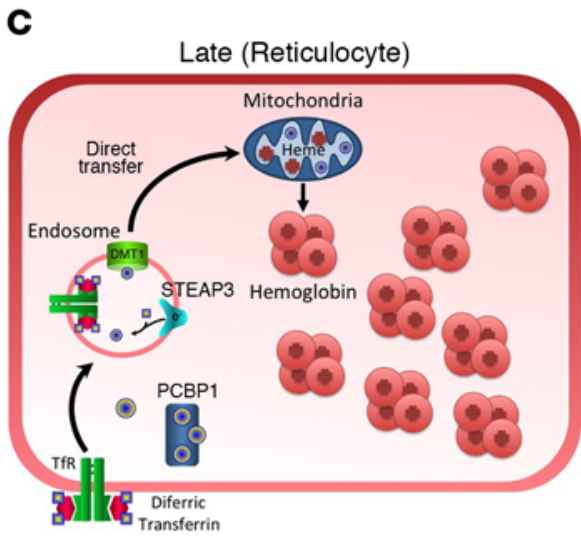

Heme and globin synthesis

Figure 8. Model of changing paths of iron flux in developing erythrocytes. (A) In earliest stages of terminal differentiation, proerythroblasts upregulate transferrin receptor (TfR) and begin accumulating iron. Iron is bound by PCBP1 and delivered to ferritin. Turnover of ferritin is low; heme and Hb synthesis have not begun. (B) At the middle stages of development, iron is delivered to ferritin and NCOA4 captures ferritin and directs it into the autophagosome, which fuses with the lysosome. Lysosomal iron is delivered to mitochondria by an undetermined mechanism that could involve direct transfer. Heme and Hb synthesis are high. Baso e., basophilic erythroblast; Ortho e., orthochromatic erythroblast. Dash indicates range. (c) In late erythrocyte development, circulating reticulocytes progressively lose intracellular organelles. Iron is not directed into ferritin and may be delivered directly to mitochondria for heme synthesis.

sate for the loss of ferritin. Alternatively, PCBP1 may be involved in ferritin-independent iron trafficking that serves to directly or indirectly facilitate iron delivery to mitochondria. Further studies are needed to clarify these possible mechanisms in erythroid cells.

In addition to PCBP1, PCBP2 can deliver iron to ferritin in yeast and cultured human cells (33). Our findings from erythroid cells, however, support a different role for PCBP2 in regulating cellular iron distribution. PCBP2 was not detected in complex with ferritin in erythroid progenitors, and the loss of PCBP2 increased iron incorporation into both ferritin and heme without compromising cellular accumulation of exogenous iron, suggesting that PCBP2 serves to limit ferritin iron accumulation in these cells. Codepletion of PCBP1 and PCBP2 resulted in a complete loss of PCBP2's capacity to limit ferritin metallation, meaning that PCBP2's effects on ferritin are mediated through PCBP1. Furthermore, PCBP2 depletion enhanced the binding of PCBP1 to ferritin. Thus, PCBP2 functions to repress the PCBP1-mediated iron transfer through ferritin. How erythroid PCBP2 exerts this unique regulation on the activity of its homolog, PCBP1, warrants further investigation. In some cells, PCBP2 can be found in a heterodimeric complex with PCBP1. This complex could impede PCBP1-mediated iron delivery to ferritin in erythroid cells and redirect the iron to other cellular targets, a process that would be lost in PCBP2-depleted cells. Recent studies suggest that PCBP2 functions in the acquisition and delivery of iron to membrane transporters that mediate iron import into (39) and efflux from (40) the cytosol. PCBP2 depletion did not affect the net cellular accumulation of iron from ${ }^{55} \mathrm{Fe}_{2}-\mathrm{Tf}$ in the G1E-ER4 cells; however, the specific contribution of PCBP2 to uptake or efflux of iron in these cells requires further investigation.

Our studies have identified iron flux through ferritin as an important step in the delivery of iron to the mitochondria for heme synthesis in early stages of terminal erythroid differentiation. Degradation of ferritin in the lysosome will effect the release of iron into the lumen of the lysosome, but the mechanism by which this iron is delivered to the mitochondria requires further investigation. Transfer of lysosomal iron into the cytosolic labile iron pool would seem to be counterproductive and expose the cell to the toxic effects of iron. A direct transfer mechanism has been proposed to move iron from the endosomal compartment to mitochondria in developing reticulocytes $(9,10)$. It is tempting to speculate that a similar mechanism exists to transfer lysosomal iron to mitochondria, perhaps via direct contact between organellar membranes, as has been observed in yeast and melanocytes (41).

\section{Methods}

Cell lines, siRNA-mediated knockdown, and iron treatment. G1E-ER4 cells were maintained in Iscove's modified Dulbecco's medium (IMDM) with 15\% FBS, $100 \mathrm{U} / \mathrm{ml}$ penicillin-streptomycin, $2 \mathrm{U} / \mathrm{ml}$ EPO (Amgen Epogen), monothioglycerol (1:10,000), and conditioned medium from Kit ligand-producing CHO cells (1:200). For differentiation, cells at $2 \times 10^{5}$ cells $/ \mathrm{ml}$ were cultured for up to 48 hours after adding $100 \mathrm{~nm} \beta$-estradiol. For RNA interference, siRNA was delivered via the Nucleofector II device (Lonza) as previously described (28). Briefly, G1E-ER4 cells $\left(6 \times 10^{6}\right.$ per transfection) were transfected twice with 240 or 480 pmoles of siRNA, with a 24 -hour interval. Upon the second transfection, cells were plated and induced for differentiation at conditions above. All knockdown experiments were carried out using Silencer Select siRNA for mouse genes as identified: s76739 for PCBP1, s71297 for PCBP2, s77517 for NCOA4 (Ambion). Negative control siRNA (Ambion 4390847) was used for nontargeting transfections. Ferric ammonium citrate was added to cell cultures as supplemental iron at a final concentration of $100 \mu \mathrm{g} / \mathrm{ml}$.

Animals and tissue collection. Wild-type (C57BL/6), CMV-Cre (B6.C-Tg[CMV-Cre]1Cgn/J) and Cre-ER ${ }^{\mathrm{T}}$ (B6.Cg-Tg[CAG-cre/Esr1] $5 \mathrm{Amc} / \mathrm{J}$ ) mice were purchased from The Jackson Laboratory. Targeting vectors and mice with a floxed allele of $P c b p 1$ were generated by InGenious Targeting Laboratory. Targeted C57BL/6-129SvEv hybrid embryonic stem cells were microinjected into C57BL/6 blastocysts. Resulting chimeras with a high-percentage agouti coat color were mated to C57BL/6 FLP mice to remove the FRT-flanked neo selection cassette. Mice carrying floxed alleles of $P c b p 1$ were backcrossed for 4 to 6 generations with $\mathrm{C} 57 \mathrm{BL} / 6$ mice. $P c b p 1^{f / f l}$ males were crossed with 
$P c b p 1^{f / f l} C r e-E R^{T(+/)}$ females to produce male offspring of Pcbp $1^{f / f l}$ and $P c b p 1^{f / f l} C r e-E R^{T(+)-)}$ genotypes. Mice were weaned on a diet supplemented with $500 \mathrm{mg} / \mathrm{kg}$ tamoxifen (Envigo) for Cre activation. Animals were euthanized by $\mathrm{CO}_{2}$, and blood was collected using heparin-pretreated syringes and tubes via cardiac puncture. The liver and kidney were excised and placed in RNAlater (Ambion) for preservation. Marrow cells were ejected from the femur and tibia with icecold PBS, pooled, and immediately processed for protein or RNA preparations. Ter $119^{+}$erythroid cells were isolated from bone marrow cells via MACS cell separation using anti-Ter-119 MicroBeads (Miltenyi Biotec 130-049-901). Genotyping was performed by PCR reactions of tail DNA using the MyTaq Extract-PCR Kit (Bioline). Primers for identifying wild-type, floxed, and null alleles of $P c b p 1$ are listed in Supplemental Table 2.

Primary erythroid cell preparation and culture. Primary erythroid progenitors were prepared from animals fed a tamoxifen diet for more than 4 weeks. Acute hemolytic anemia was produced by i.p. injections of PHZ in saline $(60 \mathrm{mg} / \mathrm{kg}$ body weight) on days 0 and 1 . Spleens were harvested on day 4 and gently mashed on and passed through a $100-\mu \mathrm{m}$ cell strainer with chilled PHZ culture medium (alpha modification of Eagle's medium [AMEM] containing 10\% FBS and $100 \mathrm{U} / \mathrm{ml}$ penicillin-streptomycin). Mature rbc were removed using ammoniumchloride-potassium (ACK) lysis buffer. After washes with PHZ culture medium, recovery and viability of nucleated erythroid progenitors were assessed using an automated cell counter (Invitrogen) with trypan blue exclusion. For ex vivo erythroid maturation, the isolated splenocytes were plated at $5 \times 10^{5}$ cells $/ \mathrm{ml}$ and treated with EPO at $2 \mathrm{U} / \mathrm{ml}$. 4-Hydroxytamoxifen (50 nM; Sigma-Aldrich) was added to sustain Cre recombinase activity ex vivo.

Western analyses and co-IP. G1E-ER4 cells or erythroid splenocytes were lysed in a buffer containing $100 \mathrm{mM}$ Tris- $\mathrm{HCl}$ (pH 7.5), $150 \mathrm{mM}$ $\mathrm{NaCl}, 0.1 \%$ Igepal CA-630, 5\% glycerol, and an EDTA-free protease inhibitor cocktail (Roche). Bone marrow lysates were prepared using 1× RIPA buffer (MitoSciences) supplemented with the above protease inhibitors. After clarification by centrifugation, protein contents were determined using the BCA method (Pierce). For Western blots, equal amounts of proteins were boiled in $1 \times$ LDS containing $2.5 \% 2-$ mercaptoethanol (BME) separated by $4 \%-12 \%$ Bis-Tris PAGE in 2-(Nmorpholino)ethanesulfonic acid (MES) SDS running buffer (Invitrogen) and transferred to nitrocellulose membrane using the iBlot System (Invitrogen). Effective transfer was confirmed by Ponceau staining. Immunoblotting was carried out using the following primary antibodies and dilutions: anti-ferritin at 1:2,000 (Sigma-Aldrich, catalog F5012); antiPCBP1 at 1:5,000 (ref. 16); anti-PCBP2 at 1:500 (Abnova, M07, clone 5F12); anti-NCOA4 at 1:1,000 (Bethyl Laboratories, catalog A302-272A); anti- $\beta$-globin at 1:200 (Santa Cruz Biotechnology Inc., catalog sc-22718); anti-IRP2 at 1:1,000 (clone UT-30, gift from Elizabeth Leibold); antiBACH1 at 1:1,000 (clone A1-6, gift from Mitsuyo Matsumoto); anti- $\alpha$ tubulin at 1:10,000 (Abcam, catalog ab7291); and anti- $\beta$-actin, 1:5,000 (Abcam, catalog ab8224). Proteins were visualized and quantified using relevant IRDye-conjugated secondary antibodies and an ODYSSEY CLx infrared imager (LiCor). For isolation of ferritin and its bound protein, equal amounts of protein were incubated with the aforementioned ferritin antibodies precoupled to Dynabeads Protein A (Life Technologies). Immune complexes were captured by magnet, extracted from beads by boiling in $2 \times$ LDS buffer plus $5 \%$ BME, and processed for Western blot analyses as described above. Nonspecific rabbit IgG (Santa Cruz Bio- technology Inc., sc-2027) was used for control IP, and post-IP supernatants were analyzed to assure complete immunodepletion of ferritin.

Real-time PCR. RNAlater-treated (Ambion) organs were disrupted using a rotor-stator homogenizer (Omni International), while bone marrow, splenocytes, and G1E-ER4 cells were lysed via repeated pipetting in TRI Reagent (Ambion). RNA was isolated using the RiboPure Kit (Ambion) and reverse transcribed for 2-step SYBR green realtime PCR (ABI). Pcbp1, Pcbp2, Ncoa4, Tfrc, Hbb-b1, and Actb transcript abundance was assessed using QuantiTect primer assays (QIAGEN). A primer set for simultaneous detection of $\mathrm{Hba}-\mathrm{a} 1$ and $\mathrm{Hba}-\mathrm{a} 2 \mathrm{mRNA}$ was designed from a sequence conserved between the 2 transcripts using NCBI's Primer-BLAST (https://www.ncbi.nlm.nih.gov/tools/ primer-blast/): Hba, forward, 5'-CGTGCTGACCTCCAAGTACC-3'; Hba, reverse, 5'-GGTACAGGTGCAAGGGAGAG-3'. Alas2, Epo, Hamp, and Erfe products were amplified via primers reported elsewhere (35, $42,43)$. Specificity of each assay was confirmed by amplicon melt curves, and data were analyzed using the double $\Delta \mathrm{Ct}$ method.

Radiolabeled iron assays. ${ }^{55} \mathrm{Fe}$-laden $\mathrm{Tf}$ was prepared by saturation of apo-Tf with ${ }^{55} \mathrm{FeCl}_{2}$ complexed to nitrilotriacetic acid as previously described (44). ${ }^{55} \mathrm{Fe}_{2}$-Tf was added to erythroid cell cultures at a physiological concentration, $2.6 \mu \mathrm{M}$, upon induction of development. Radiolabeled cells were collected and washed with ice-cold PBS containing $10 \mathrm{mM}$ EDTA and kept in chilled PBS. A part of cell suspension was collected for total cell ${ }^{55} \mathrm{Fe}$ measures and protein quantitation. Heme fractions were prepared by an acidified organic solvent extraction method previously reported (17). ${ }^{55} \mathrm{Fe}$ contents were determined by scintillation counting and normalized to cellular protein levels from BCA assays. For assessment of ${ }^{55} \mathrm{Fe}$ incorporated into ferritin cages and $\mathrm{Hb}$, radiolabeled cells were lysed in $20 \mathrm{mM}$ Tris- $\mathrm{HCl}$ (pH 7.5), $40 \mathrm{mM} \mathrm{KCl,} \mathrm{0.5 \%} \mathrm{Triton} \mathrm{X-100,} \mathrm{and} \mathrm{an} \mathrm{EDTA-}$ free protease inhibitor cocktail (Roche). Equal amounts of protein were separated in $50 \mathrm{mM}$ Tris- $\mathrm{HCl}(\mathrm{pH} 7.5), 0.02 \%$ bromophenol blue, and $5 \%$ glycerol by $3 \%-8 \%$ Tris-Acetate PAGE with TrisGlycine native running buffer (Invitrogen). Gels were dried under vacuum and exposed to a Storage Phosphor screen (GE Healthcare) for visualization by a Typhoon TRIO imager (Amersham Biosciences). Radio-iron signals were quantified via densitometric analyses using ImageQuant TL software (GE Healthcare).

CBC, total cellular heme, and plasma iron assays. CBC profiles of heparinized blood were assessed using a ProCyte Dx Hematology Analyzer (IDEXX). Heme contents in cell lysates were measured using the QuantiChrom Heme Assay Kit (BioAssay Systems) according to the manufacturer's instructions. A ferrozine chromogenic method with iron standard solutions (Fisher Scientific) determined the total plasma iron levels as described elsewhere (45).

Histology. Wright-Giemsa staining of blood smears and cytospins of G1E-ER4 cells were carried out at the Department of Laboratory Medicine of the NIH Clinical Center. Bone marrow slides were prepared from femurs fixed in $10 \%$ buffered formalin and decalcified by $5 \%$ EDTA. Tissue sectioning and H\&E staining were performed at American Histo Labs Inc. Images of stained slides were produced using a NanoZoomer Digital Pathology system (Hamamatsu). Apoptotic cells within the marrow were identified using the In situ BrdU-Red DNA Fragmentation (TUNEL) Assay kit (Abcam).

Statistics. Data are presented as mean \pm SEM of the indicated $n$ of independent cell culture experiments or animals. Statistical significance was determined using paired or unpaired Student's $t$ tests, 
repeated measures of ANOVA, or 2-way ANOVA with Bonferroni's or Dunnett's post hoc tests, depending on the study design and number of comparisons (Prism 5.0a, GraphPad Software). Significance was set at $P$ $<0.05$, 2-sided, for all analyses.

Study approval. All animal studies were reviewed and approved by the NIDDK Animal Care and Use Committee and performed in compliance with NIH guidelines for the humane care of animals.

\section{Author contributions}

MSR conceived and designed the research studies, conducted experiments, acquired and analyzed data, prepared figures, and wrote the manuscript. DZ designed and conducted experiments and acquired and analyzed data. OP designed and conducted experiments, acquired and analyzed data, prepared figures, and wrote the manuscript. MSE conducted experiments and acquired data. CCP conceived and designed the research studies and wrote the manuscript.

\section{Acknowledgments}

We thank Mitchell Weiss (St. Jude Children's Research Hospital, Memphis, Tennessee, USA) for the gift of G1E-ER4 and CHO-KL cell lines. We thank Elizabeth Leibold (University of Utah, Salt Lake City, Utah, USA) and Mitsuyo Matsumoto (Tohoku University, Sendai, Japan) for antibodies against IRP2 and BACH1, respectively. These studies were supported by the Intramural Research Program of NIDDK, NIH, and the Office of Dietary Supplements, Office of the Director, NIH.

Address correspondence to: Caroline C. Philpott, Genetics and Metabolism Section, Liver Diseases Branch, NIDDK, NIH, Building 10, Room 9B-16, 10 Center Drive, Bethesda, Maryland 20892-1800, USA. Phone: 301.435.4018; E-mail: carolinep@intra.niddk.nih.gov.

MSR's present address is: Department of Food Science and Nutrition, University of Minnesota, St. Paul, Minnesota, USA.
1. McLean E, Cogswell M, Egli I, Wojdyla D, de Benoist B. Worldwide prevalence of anaemia, WHO Vitamin and Mineral Nutrition Information System, 1993-2005. Public Health Nutr. 2009;12(4):444-454.

2. Stoltzfus RJ. Iron deficiency: global prevalence and consequences. Food Nutr Bull. 2003;24(4 Suppl):S99-103.

3. Palis J. Primitive and definitive erythropoiesis in mammals. Front Physiol. 2014;5:3.

4. Ganz T, Nemeth E. Iron metabolism: interactions with normal and disordered erythropoiesis. Cold Spring Harb Perspect Med. 2012;2(5):a011668.

5. Theil EC. Ferritin: the protein nanocage and iron biomineral in health and in disease. Inorg Chem. 2013;52(21):12223-12233.

6. Konijn AM, Hershko C, Izak G. Ferritin synthesis and iron uptake in developing erythroid cells. Am J Hematol. 1979;6(4):373-379.

7. Beaumont C, Dugast I, Renaudie F, Souroujon M, Grandchamp B. Transcriptional regulation of ferritin $\mathrm{H}$ and $\mathrm{L}$ subunits in adult erythroid and liver cells from the mouse. Unambiguous identification of mouse ferritin subunits and in vitro formation of the ferritin shells. J Biol Chem. 1989;264(13):7498-7504.

8. Grasso JA, Hillis TJ, Mooney-Frank JA. Ferritin is not a required intermediate for iron utilization in heme synthesis. Biochim Biophys Acta. 1984;797(2):247-255.

9. Zhang AS, Sheftel AD, Ponka P. Intracellular kinetics of iron in reticulocytes: evidence for endosome involvement in iron targeting to mitochondria. Blood. 2005;105(1):368-375.

10. Sheftel AD, Zhang AS, Brown C, Shirihai OS, Ponka P. Direct interorganellar transfer of iron from endosome to mitochondrion. Blood. 2007;110(1):125-132.

11. Darshan D, Vanoaica L, Richman L, Beermann F, Kühn LC. Conditional deletion of ferritin $\mathrm{H}$ in mice induces loss of iron storage and liver damage. Hepatology. 2009;50(3):852-860.

12. Speyer BE, Fielding J. Ferritin as a cytosol iron transport intermediate in human reticulocytes. Br J Haematol. 1979;42(2):255-267.

13. Vaisman B, Fibach E, Konijn AM. Utilization of intracellular ferritin iron for hemoglobin synthesis in developing human erythroid precursors. Blood. 1997;90(2):831-838.

14. Makeyev AV, Liebhaber SA. The poly(C)-binding proteins: a multiplicity of functions and a search for mechanisms. RNA. 2002;8(3):265-278.

15. Chkheidze AN, Lyakhov DL, Makeyev AV, Morales J, Kong J, Liebhaber SA. Assembly of the alpha-globin mRNA stability complex reflects binary interaction between the pyrimidinerich 3' untranslated region determinant and poly (C) binding protein alphaCP. Mol Cell Biol. 1999;19(7):4572-4581.

16. Shi H, Bencze KZ, Stemmler TL, Philpott CC. A cytosolic iron chaperone that delivers iron to ferritin. Science. 2008;320(5880):1207-1210.

17. Frey AG, et al. Iron chaperones PCBP1 and PCBP2 mediate the metallation of the dinuclear iron enzyme deoxyhypusine hydroxylase. Proc Natl Acad Sci USA. 2014;111(22):8031-8036.

18. Nandal A, et al. Activation of the HIF prolyl hydroxylase by the iron chaperones PCBP1 and PCBP2. Cell Metab. 2011;14(5):647-657.

19. Mancias JD, Wang X, Gygi SP, Harper JW, Kimmelman AC. Quantitative proteomics identifies NCOA4 as the cargo receptor mediating ferritinophagy. Nature. 2014;509(7498):105-109.

20. Dowdle WE, et al. Selective VPS34 inhibitor blocks autophagy and uncovers a role for NCOA4 in ferritin degradation and iron homeostasis in vivo. Nat Cell Biol. 2014;16(11):1069-1079.

21. Mancias JD, et al. Ferritinophagy via NCOA4 is required for erythropoiesis and is regulated by iron dependent HERC2-mediated proteolysis. Elife. 2015;4:e10308.

22. Nilsson R, et al. Discovery of genes essential for heme biosynthesis through large-scale gene expression analysis. Cell Metab. 2009;10(2):119-130.

23. Galloway JL, Wingert RA, Thisse C, Thisse B, Zon LI. Combinatorial regulation of novel erythroid gene expression in zebrafish. Exp Hematol. 2008;36(4):424-432.

24. Bellelli R, et al. NCOA4 Deficiency Impairs Systemic Iron Homeostasis. Cell Rep. 2016;14(3):411-421.

25. Yeh S, Chang C. Cloning and characterization of a specific coactivator, ARA70, for the androgen receptor in human prostate cells. Proc Natl Acad Sci USA. 1996;93(11):5517-5521.

26. Heinlein CA, Ting HJ, Yeh S, Chang C. Identification of ARA70 as a ligand-enhanced coactivator for the peroxisome proliferatoractivated receptor gamma. J Biol Chem. 1999;274(23):16147-16152.

27. Bellelli R, et al. NCOA4 transcriptional coactivator inhibits activation of DNA replication origins. Mol Cell. 2014;55(1):123-137.

28. Lee HY, Johnson KD, Boyer ME, Bresnick EH. Relocalizing genetic loci into specific subnuclear neighborhoods. J Biol Chem. 2011;286(21):18834-18844.

29. Welch JJ, et al. Global regulation of erythroid gene expression by transcription factor GATA-1. Blood. 2004;104(10):3136-3147.

30. Anderson CP, Shen M, Eisenstein RS, Leibold EA. Mammalian iron metabolism and its control by iron regulatory proteins. Biochim Biophys Acta. 2012;1823(9):1468-1483.

31. Zenke-Kawasaki Y, et al. Heme induces ubiquitination and degradation of the transcription factor Bach1. Mol Cell Biol. 2007;27(19):6962-6971.

32. Tahara T, Sun J, Igarashi K, Taketani S. Hemedependent up-regulation of the alpha-globin gene expression by transcriptional repressor Bach1 in erythroid cells. Biochem Biophys Res Commun. 2004;324(1):77-85.

33. Leidgens $S$, et al. Each member of the poly-r(C)binding protein 1 (PCBP) family exhibits iron chaperone activity toward ferritin. J Biol Chem. 2013;288(24):17791-17802.

34. Ghanem LR, et al. The Poly $(\mathrm{C})$ Binding Protein Pcbp2 and Its Retrotransposed Derivative Pcbp1 Are Independently Essential to Mouse Development. Mol Cell Biol. 2015;36(2):304-319.

35. Kautz L, Jung G, Valore EV, Rivella S, Nemeth E, Ganz T. Identification of erythroferrone as an erythroid regulator of iron metabolism. Nat Genet. 2014;46(7):678-684.

36. Andriopoulos B, et al. BMP6 is a key endogenous regulator of hepcidin expression and iron metabolism. Nat Genet. 2009;41(4):482-487.

37. Hara H, Ogawa M. Erthropoietic precursors in 
mice with phenylhydrazine-induced anemia. $A m$ JHematol. 1976;1(4):453-458.

38. Griffiths RE, et al. Maturing reticulocytes internalize plasma membrane in glycophorin A-containing vesicles that fuse with autophagosomes before exocytosis. Blood. 2012;119(26):6296-6306.

39. Yanatori I, Yasui Y, Tabuchi M, Kishi F. Chaperone protein involved in transmembrane transport of iron. Biochem J. 2014;462(1):25-37.

40. Yanatori I, Richardson DR, Imada K, Kishi F. Iron export through the transporter ferroportin 1 is modulated by the iron chaperone PCBP2. J Biol
Chem. 2016;291(33):17303-17318.

41. Daniele T, Schiaffino MV. Lipid transfer and metabolism across the endolysosomalmitochondrial boundary. Biochim Biophys Acta. 2016;1861(8 Pt B):880-894.

42. Ghosh MC, et al. Deletion of iron regulatory protein 1 causes polycythemia and pulmonary hypertension in mice through translational derepression of HIF2 $\alpha$. Cell Metab. 2013;17(2):271-281.

43. Ryu MS, Lichten LA, Liuzzi JP, Cousins RJ. Zinc transporters ZnT1 (Slc30a1), Zip8 (Slc39a8), and Zip10 (Slc39a10) in mouse red blood cells are differentially regulated during erythroid development and by dietary zinc deficiency. J Nutr. 2008;138(11):2076-2083.

44. Inman RS, Wessling-Resnick M. Characterization of transferrin-independent iron transport in K562 cells. Unique properties provide evidence for multiple pathways of iron uptake. J Biol Chem. 1993;268(12):8521-8528.

45. Gosriwatana I, Loreal O, Lu S, Brissot P, Porter J, Hider RC. Quantification of non-transferrinbound iron in the presence of unsaturated transferrin. Anal Biochem. 1999;273(2):212-220. 